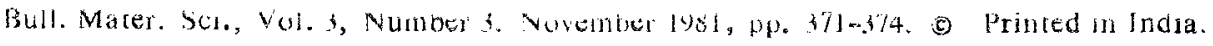

\title{
Characterisation of high purity rare-earth oxides by x-ray fluorescence methods
}

\author{
R M DIXIT \\ Spectroscopy Division, Bhabha Atomic Research Centre, Trombay, \\ Bombay 400 085, India
}

MS Received 23 March 1981

\begin{abstract}
X-ray fluorescence methods for the determination of individual rare earth impurities in high purity rare earth oxides viz., $\mathrm{Y}_{2} \mathrm{O}_{3}, \mathrm{La}_{2} \mathrm{O}_{3}, \mathrm{CeO}_{2}$, $\mathrm{Pr}_{6} \mathrm{O}_{11}, \mathrm{Nd}_{2} \mathrm{O}_{3}, \mathrm{Sm}_{2} \mathrm{O}_{3}, \mathrm{Eu}_{2} \mathrm{O}_{3}, \mathrm{Gd}_{2} \mathrm{O}_{3}, \mathrm{~Tb}_{4} \mathrm{O}_{7}, \mathrm{Dy}_{2} \mathrm{O}_{3}, \mathrm{HO}_{2} \mathrm{O}_{3}$ and $\mathrm{Er}_{2} \mathrm{O}_{3}$ have been developed in our laboratory. The samples are converted to oxalate form and doutle-layer pellets are prepared using boric acid as binding material. A semiautomatic $x$-ray spectrometer is used for the analyses. Choice of instrumental parameters and analysis lines is discussed. The determination range varies from $0.005 \%$ to $1.0 \%$ for most elements with good precision and adequate accuracy.
\end{abstract}

keywerds. Rare earth; $x$-ray fluorescence.

\section{Introduction}

High purity rare earths are finding extensive use in industry and technology. Rapid and accurate analysis of these materials for individual rare earth impurities is therefore quite important. Optical emission spectroscopic (OES) methods have generally been used for the analysis in our laboratory. But these methods suffer from line interferences due to rich line spectra of rare earth. elements and hence they are limited to a few rare earth elements in each rare warth matrix.

Some optical luminiscence methods viz., x-ray excited optical fluorescence (XEOF) and UV excited optical luminiscence, are applicable only to a few rare earth oxide matrices, which form suitable host to support fluorescence and also are prone to concentration-dependant inter-element effects even at total impurity concentration of about $100 \mathrm{ppm}$.

$\mathrm{X}$-ray fluorescence (XRF) methods are ideally suited for this analysis due to simple $x$-ray spectra of rare earth elements and rapidity, high precision and adequate accuracy of the XRF methods.

Lytle and Heady (1959) have developed XRF methods for the analysis of individual rare earth elements in $\mathrm{Y}_{2} \mathrm{O}_{3}, \mathrm{CeO}_{2}, \mathrm{Pr}_{6} \mathrm{O}_{11}, \mathrm{Nd}_{2} \mathrm{O}_{3}$ and $\mathrm{Sm}_{2} \mathrm{O}_{3}$ using fjxed count method. For high purity rare earths with impurities at trace levels the counting times are as high as 10 minutes. They have also used packed powders in sample cell for sample presentation which is obviously not reproducible. 
The XRF methods developed in our laboratory (Chandola et al 1976a b, Chandola and Mohile 1977, 1978 and Dixit and Deshpande 1975, 1976, 1977a, b, 1978; Dixit and Kapoor 1980) use fixed time counting (maximum $100 \mathrm{sec}$ ) and reproducible double-layer pellet method for sample preparation and therefore have better precision, lower estimation limit with adequate accuracy.

\section{Experimental procedure}

\subsection{Preparation of standards and samples}

Standards containing known amounts of rare earth elements in high purity rare earths are obtained by mixing the solutions of rare earth oxides in nitric acid in appropriate quantities and then precipitating them as oxalates. Samples are also converted to oxalate form similarly to remove the previous history of sample if any. The rare earth standards/samples in oxalate form are mixed with pure boric acid as binder in different ratios. The rare earth oxalate boric acid mixture is pressed over a primary boric acid pellet as a double layer. The amount of sample required is determined experimentally to give 'infinite thickness' for L lines of rare earth elements.

\subsection{Choice of instrumental parameters}

A semiautomatic X-ray spectrometer (Philips PW 1220) coupled with ultrastable generator PW 1140 is used. The samples in the form of double layer pellets are exposed to primary $x$-ray; from tungsten target $x$-ray tube. The characteristic $\mathrm{L} x$-ray lines of tungsten viz., $\mathrm{L}_{\alpha_{1}} \lambda=1.476 \AA$ and $\mathrm{L}_{\beta_{1}} \lambda=1.252 \AA$ are useful in exciting rare earth $L$ spectra. The $x$-ray tube is operated at $60 \mathrm{kV}-35 \mathrm{~mA}$ to give optimum signal to noise ratio. $\mathrm{LiF}(200)$ with $2 \mathrm{~d}=4.028 \AA$ and very high reflectivity is found to be most suitable for the purpose. Pulse height selection is used to minimise higher order interferences. The instrumental parameters are summarised in table 1.

Table 1. Instrumental parameters.

X-ray Spectrometer

$X$-ray generator

X-ray tube target power

Power

Analysing crystals

Collimater

Detector

Counting

Analysis lines

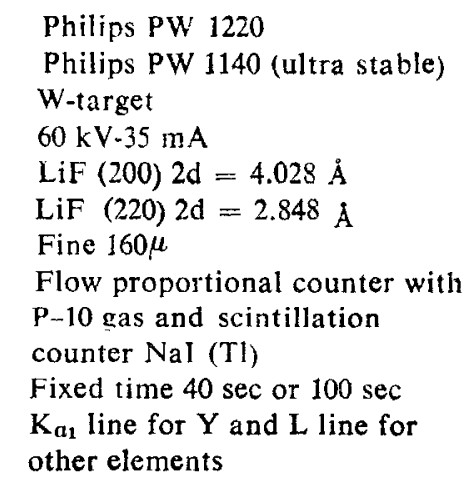




\subsection{Analysis procedure}

A blank, a standard and samples are loaded in four sample positions. These samples are exposed to primary $x$-rays sequentially. The intensities of fluorescent $x$-rays after diffraction through analysing crystal $\mathrm{LiF}$ (200) at appropriate $2 \theta$ angles are measured. The net peak intensity is plotted against known concentrations to get a working curve which is used for determination of unknown concentrations in samples.

\section{Results and discussion}

\subsection{Choice of analysis lines}

An $x$-ray $L$ line either $L_{a_{1}}$ or $L_{\beta_{1}}$ with least interference is chosen for analysis: The line overlap corrections are carried out wherever partial interference is present. Typical interference problems faced in this analysis are as follows.

$Y_{2} \mathrm{O}_{3}$ matrix: $\mathrm{HoL}_{\alpha_{1}}$ has interference from $\mathrm{GdL}_{\beta_{1}}$ which is also one of the common impurities. $\mathrm{HoL}_{\beta_{1}}$ has a high background due to the presence of $\mathrm{YK}_{\alpha}$ 2nd order line. $\mathrm{HoL}_{\beta_{1}}$ is preferred though it gives poor sensitivity.

$\mathrm{La}_{2} \mathrm{O}_{3}$ matrix: $\operatorname{PrL}_{\alpha_{1}}(\lambda=2.463 \AA)$ has an interference from matrix line $\operatorname{LaL}_{\beta_{1}}(\lambda=2.458 \AA)$. $\operatorname{Pr} L_{\beta_{1}}(\lambda=2.258 \AA)$ and $\operatorname{PrL}_{\beta_{2}}(\lambda=2.119 \AA)$ are strongly absorbed by lanthanum matrix itself due to absorption at $\operatorname{L}$ III $(\lambda=2.259 \AA)$ and $L I(\lambda=1.973 \AA)$, PrI $L_{-\gamma_{1}}$, line is too weak to be considered for analysis line. $\operatorname{PrL}_{\beta_{1}}$ has been chosen for analysis line, though it gives poor sensitivity.

$\mathrm{Gd}_{2} \mathrm{O}_{3}$ matrix: $\mathrm{YbL}_{\alpha_{1}}$ has partial interference from $\mathrm{TbL}_{\beta_{2}}$ and $\mathrm{DyL}_{\beta_{3}}$ $\operatorname{LiF}(220) 2 \mathrm{~d}=2.848 \AA$ has been used to resolve the lines and minimise the above interference.

$\mathrm{Dy}_{2} \mathrm{O}_{3}$ matrix: Er $\mathrm{L}_{\alpha_{1}}$ has an interference from $\mathrm{TbL}_{\beta_{3}}$ and $\mathrm{GdL}_{\beta_{2}} \mathrm{LiF}(220)$ crystal dong with line overlap corrections are used for analysis.

The analysis lines chosen for different rare earth elements in various matrices are given in references mentioned earlier.

\subsection{Precision and accuracy}

The precision of the methods is determined by taking 15 values for each element in each standard in three sets of five observations each. The percent standard deviation varied from $40 \%$ to $0.1 \%$ depending upon the standard, element and matrix.

The accuracy of the methods was tested by analysing synthctic samples. It was found that the accuracy was quantitutive within the experimental errors. 


\section{Acknowledgement}

The author is grateful to Dr N A Narasimham for his keen interest and to Shri L C Chandola for some useful suggestions.

\section{References}

Chandola L C, Machado I J and Mohile A N 1976a J. Radio Anal. Chem. 34389

Chandola L C, Machado I J and Mohile A N 1976 Mikro. Chim. Acta 11399

Chandola L C and Mohile A N 1977 Curr. Sci. 46299

Chan dola L C and Mohile A N 1978 Indian. J. Pure Appl. Phys. 16851

Dixit R M and Deshpande S S 1975 An XRF method for determination of common rare earth impurities in high purity yttrium oxide Report BARC 897

Dixit R M and Deshpande S S 1976 Chem. Anal. (Warsaw) 211353

Dixit R M and Deshpande S S 1977a Fresenius Z. Anal. Chem. 287132

Dixit R M and Deshpande S S 1977b Fresenius $Z$. Anal. Chem. 238 18J

Dixit R M and Deshpande S S 1978 Fresenitis Z. Anal. Chem. 292375

Dixit R M and Kappor S K 1979 Fresenius Z. Anal. Chicm. 296394

Dixit R M and Deshpande S S 1980 Fresenius $Z$. Anal. Chem. 303111

Lytle F W and Heady H H 1959 Anal. Chem. 31809 